\title{
Politique
}

\section{L'orientation interventionniste des idéologies des partis politiques québécois depuis 1970}

\section{Réjean Landry}

Numéro 13, printemps 1988

Crise de décision

URI : https://id.erudit.org/iderudit/040584ar

DOI : https://doi.org/10.7202/040584ar

Aller au sommaire du numéro

Éditeur(s)

Société québécoise de science politique

ISSN

0711-608X (imprimé)

1918-6584 (numérique)

Découvrir la revue

Citer cet article

Landry, R. (1988). L'orientation interventionniste des idéologies des partis politiques québécois depuis 1970. Politique, (13), 63-85.

https://doi.org/10.7202/040584ar d'utilisation que vous pouvez consulter en ligne.

https://apropos.erudit.org/fr/usagers/politique-dutilisation/ 


\title{
L'ORIENTATION INTERVENTIONNISTE DES IDÉOLOGIES DES PARTIS POLITIQUES QUÉBÉCOIS DEPUIS 1970*
}

\author{
par \\ Réjean Landry \\ Université Laval
}

Les idéologies politiques de la scène québecoise ont été scrutées et analysées essentiellement suivant deux démarches. Plusieurs spécialistes des sciences sociales (Rioux, 1968; Dion, 1975; Monière 1977) ont adopté une perspective macroscopique qui vise principalement à décrire et à expliquer les changements qualitatifs dans les idéologies selon une méthode bien explicitée par Monière $(1977$, 9), qui consiste «à relire la littérature sociologique, à en tirer des informations qui (...) semblent pertinentes, à organiser cette matière et, à l'occasion, à soulever des hypothèses exploratoires ou à souligner certaines contradictions ». À cette perspective macroscopique et qualitative, quelques politistes ont préféré opter pour une démarche plus microscopique, en procédant à une analyse

* Cette recherche a été réalisée grâce à une subvention du Conseil de recherche en sciences humaines du Canada. En outre, l'auteur tient aussi à remercier Paule Duchesneau, François Michaud pour leur participation à la préparation de ce manuscrit et trois évaluateurs anonymes de la revue Politique pour leurs commentaires et leurs critiques de la première version du manuscrit de cet article.

Politique, 13 (Printemps 1988). 
quantitative comparée des idéologies des partis politiques (Mellos, 1970; Latouche 1976; Pelletier, 1980).

Mellos, Latouche et Pelletier postulent que les programmes officiels des partis représentent de façon condensée leurs idéologies. Il importe de reconnaître d'emblée que les programmes officiels ne constituent pas les seuls documents produits par les partis. Les dirigeants des partis émettent des communiqués de presse, prononcent des discours, accordent des entrevues aux médias et publient parfois des livres. Un certain nombre de ces manifestations correspondent à la position d'individus plutôt qu'à la position officielle des partis. Les programmes revêtent toutefois une caractéristique unique, ils représentent la position officielle des partis, c'est-à-dire l'énoncé de position du parti plutôt que celui d'un individu. Si les programmes officiels atteignent moins d'électeurs que d'autres manifestations telles que les discours télévisés, ils servent néanmoins de points de référence, de dernier recours pour les politiciens aussi bien que pour les commentateurs et les analystes de la scène politique (Pomper, 1980; Budge et Farlie, 1983; Rose, 1984).

Les programmes officiels présentent de façon condensée les interventions gouvernementales que les partis projettent de réaliser au lendemain d'une victoire électorale. Les projets d'interventions des programmes officiels comportent tous les éléments constitutifs des idéologies: ils rationalisent et légitimisent l'intervention de l'État tout en visant à mobiliser la participation et le vote des individus. Suivant cette perspective, les idéologies résument et symbolisent les différences dans les conceptions et les attitudes des partis à l'égard des interventions gouvernementales.

L'analyse des idéologies des partis ne peut toutefois se limiter à décrire les caractéristiques de leurs projets d'interventions gouvernementales; elle doit tenter d'expliquer et de prédire le rapport qui existe entre le discours des partis et leurs intérêts électoraux. L'examen de ce rapport a donné naissance à deux explications 
principales: la confrontation directe et l'accentuation sélective. Suivant la première explication, les partis entrent en compétition pour l'obtention des votes en offrant des interventions différentes sur les mêmes enjeux, tandis que, d'après la seconde explication, la compétition pour l'obtention des votes s'effectue principalement en accentuant l'importance que chacun des partis accorde à différents enjeux d'interventions gouvernementales (Budge et Farlie, 1983). Cette seconde explication signifie que les partis peuvent s'ignorer les uns les autres parce que, contrairement à des équipes de hockey, les partis politiques ne sont pas forcés de s'affronter au même moment sur une même patinoire. Bref, rien ne force les partis à prendre position au même moment sur les mêmes enjeux.

La théorie des choix collectifs, particulièrement les modèles spatiaux de compétition reposent à peu près exclusivement sur l'hypothèse de la confrontation directe (Downs, 1957; Barry, 1970; Riker et Ordeshook, 1973; Ordeshook, 1976; Budge et Farlie, 1976, 1978, 1983). Ils peuvent aussi servir à examiner l'hypothèse de l'accentuation sélective. Robertson (1976) et Budge et Farlie (1983a) montrent que, dans ce cas, l'analyse spatiale se fonde sur l'importance relative accordée par les partis à différents enjeux, et que certaines zones des espaces seront inoccupées puisque les partis préferent passer sous silence les enjeux qui les défavorisent pour mettre l'accent sur les enjeux qui leur procurent des avantages électoraux. Suivant cette perspective, les partis mettent l'accent sur les enjeux qui les favorisent parce qu'ils sont, dans une certaine mesure propriétaires de ces enjeux. Ainsi, le Parti libéral du Québec ne pourra jamais se prétendre plus social démocrate que le Parti québécois. La meilleure stratégie libérale consiste donc à détourner l'attention des électeurs de la nécessité de réformes sociales vers la mise en place de mesures de développement économique, chapitre à propos duquel le $\mathrm{PQ}$ ne peut prétendre avoir une meilleure crédibilité que les libéraux. 
Les deux modes de compétition, la confrontation directe et l'accentuation sélective, ne sont pas mutuellement exclusifs. L'analyse de contenu des programmes des partis politiques américains et britanniques des 50 dernières années montrent que l'hypothèse de l'accentuation sélective correspond mieux à la réalité que celle de la confrontation directe (Budge et Farlie 1983a). L'analyse comparative des programmes des partis effectuée par Latouche (1976, 149) confirme elle aussi la thèse de l'accentuation sélective:

"C'est par les thèmes traités, plutôt que par leur orientation idéologique, que les programmes des partis different surtout l'un de l'autre. On parle donc de choses différentes mais avec sensiblement la même grille idéologique.»

Les travaux de Mellos (1970), Latouche (1976) et Pelletier (1980) démontrent que l'un des enjeux idéologiques récurrents dans les programmes des partis concerne l'interventionnisme de l'État. Certains analystes soutiennent qu'au cours de la période 1960-1980, «L'État fut promu au rôle d'agent moteur principal du rattrapage». (McRoberts et Posgate, 1983, 117).

Les données de Mellos (1970, 550) montrent qu'en 1966, le PLQ favorisait davantage le développement du secteur public que du secteur privé alors que l'Union nationale tendait à faire le contraire. Recourant à une technique de cueillette et d'analyse très semblable à celle de Mellos, Latouche (1976) montre, qu'en 1973, le Parti québécois tend davantage à favoriser l'essor du secteur public (12,7\% des propositions) que du secteur privé $(5,5 \%)$ alors que le Parti libéral favorise le secteur privé $(10,0 \%)$ au détriment de l'expansion du secteur public $(8,9 \%)$.

L'analyse de contenu des programmes des partis réalisée par Pelletier (1980) arrive à des résultats similaires, bien que les techniques de cueillette et d'analyse des données soient un peu différentes. D'après ce dernier, il y aurait eu accroissement de l'interventionnisme de 1960 à 1968, c'est-à-dire augmentation du nombre de propositions favorisant l'expansion du secteur public, aussi bien du côté de l'UN que du PLQ, mais cette tendance 
aurait été plus prononcée du côté libéral que du côté unioniste. D'autre part, pendant la période subséquente, allant de 1970 à 1976, le PQ aurait fait preuve d'une plus grande propension à l'interventionnisme que le PLQ, ce dernier tendant avec le temps à devenir de moins en moins favorable à l'intervention de l'État.

Bien que l'interventionnisme de l'État soit demeuré un enjeu majeur et recurrent dans les programmes des partis aussi bien que dans les débats politiques, aucune étude empirique récente n'est venue compléter le portrait tracé par Mellos, Latouche et Pelletier. L'objet de cet article, consiste donc à effectuer une analyse comparée des propositions d'actions des programmes officiels du Parti libéral du Québec et du Parti québécois, entre 1970 et 1985, afin de dégager l'orientation de l'interventionnisme dans leurs idéologies.

La méthode de collecte des données

L'analyse de contenu des programmes des partis comprend généralement cinq étapes (Mellos, 1970; Latouche, 1976; Pelletier, 1980; Pomper, 1980; Rose, 1984; Landry et Duchesneau, 1987): la détermination de l'unité d'analyse; la sẻlection des documents à analyser; l'enregistrement des données, la vérification des données; la présentation et l'interprétation des résultats.

Les analyses de contenu des programmes des partis politiques adoptent à peu près toutes la proposition d'action ou la proposition d'intervention gouvernementale comme unité d'analyse. Les spécialistes demeurent généralement peu loquaces sur les caractéristiques intrinsèques des propositions d'actions des partis. Notre démarche méthodologique se démarque du courant dominant des travaux en définissant la proposition d'intervention gouvernementale comme un énoncé comprenant quatre éléments fondamentaux:

Un destinateur: qui énonce la proposition? Par exemple: le PLQ. 
Un verbe connecteur: Quel est le verbe d'action utilisé par le destinateur? Par exemple: mettra

L'attribut de l'action: Quel est l'attribut de l'intervention proposée par le destinateur? Par exemple: un accent particulier sur des programmes d'habitation.

Un destinataire: Quelle catégorie d'individus bénéficiera (ou supportera les coûts) de la proposition d'intervention énoncée par le destinateur? Par exemple: pour les personnes âgées.

Les documents sélectionnés pour réaliser l'analyse de contenu sont les programmes officiels du Parti libéral du Québec et du Parti québécois en vigueur au moment des élections de 1970, 1973, 1976, 1981 et 1985.

L'enregistrement des données d'une analyse de contenu est généralement réalisé à l'aide d'une fiche ou d'une grille d'analyse et d'un document déterminant les règles à suivre pour utiliser cet outil d'analyse. Mellos (1970), Latouche (1976), Pelletier (1980), Rose (1984), Budge et Farlie, (1983), ont utilisé une grille permettant de différencier les propositions d'interventions selon les thèmes abordés et suivant leur orientation en fonction de grandes dichotomies. La fiche utilisée dans notre analyse de contenu consiste en un questionnaire comprenant quatre parties: l'identification numérique de la proposition d'intervention; le domaine d'intervention visé par la proposition; les caractéristiques des coûts et des bénéfices de la proposition en fonction des destinataires; finalement, le texte de la proposition. Cette modalité d'analyse de contenu équivaut en quelque sorte à une simulation d'entrevues effectuée à l'aide d'un questionnaire fermé (Krippendorff, 1980): l'analyste pose des questions à des documents et répond à un questionnaire en suivant les consignes du guide d'utilisation de la fiche d'analyse.

L'analyse de contenu a été effectuée par une personne travaillant à temps complet. Les cas de propositions dont l'analyse soulevait 
des difficultés particulières ont été examinés sur une base hebdomadaire et leur solution avait généralement pour effet de préciser les consignes concernant l'utilisation de la fiche d'analyse. Une fois transcrites sur ordinateur les données ont été vérifiées à l'aide d'un programme informatique développé dans le but de détecter les erreurs de transcription de données (codes non prévus). Un second programme informatique a été conçu pour produire le texte des propositions pour tous les cas où des réponses sont logiquement incompatibles. Ce type de vérification était possible parce que, à la différence des fiches d'analyse qui contiennent des questions ad hoc sans liens logiques, le questionnaire utilisé pour réaliser cette étude est constitué de questions représentant les concepts d'une théorie déductive, la théorie des choix collectifs.

La lecture des cas considérés illogiques par l'ordinateur nous a amené à réviser l'analyse d'environ $4 \%$ des propositions. Voilà pour les opérations de vérification concernant la validité des données. Un troisième programme d'ordinateur a été conçu pour faire imprimer le texte des propositions en fonction des réponses aux questions de la fiche d'analyse. Les imprimés d'ordinateur de ce programme ont été vérifiés «manuellement» et ont servi de catalogues auxquels on pouvait se référer par la suite de façon à s'assurer que les propositions ayant les mêmes caractéristiques étaient toujours codées de la même façon. Ce troisième programme d'ordinateur servait donc d'outil de base pour vérifier la fiabilité de l'analyse de contenu.

\section{Présentation des résultats}

\section{L'interventionnisme de l'État}

Le degré d'interventionnisme de l'idéologie des partis politiques peut se mesurer à l'aide d'un indicateur simple: le nombre de propositions d'actions contenu dans les programmes officiels 
des partis. Le recours à cet indicateur peut être justifié par le fait que toute proposition d'action renvoie à un projet d'utilisation des ressources financières, juridiques ou symboliques de l'État.

La technique d'analyse de contenu, décrite plus haut, a permis de répertorier 6045 propositions d'actions dont 2359 sont attribuables au Parti libéral du Québec et 3686 au Parti québécois. Le nombre de projets d'actions du PLQ passe de 260 en 1970, à 389 en 1973, puis à 450 en 1976, pour atteindre le sommet de 893 en 1981, et redescendre à 367 en 1985. Du côté du PQ, le nombre de propositions grimpe de 355 à 584 , puis à 656 et à 1181, pour finalement chuter à 910 en 1985. Ces données brutes montrent donc que la tendance à l'interventionnisme des deux partis s'accroît de 1970 à 1981 pour décroître de 1981 à 1985 . Une façon simple de représenter l'évolution de l'interventionnisme est de développer un indice relatif. Un tel indice peut être calculé en comparant le nombre de propositions d'interventions de l'année 1970 , noté par $N_{t}$, au nombre d'interventions de chacune des années ultérieures, noté par $\mathrm{N}_{\mathrm{t}+\mathrm{x}}$. Ainsi l'interventionnisme du PLQ s'est accru par un facteur 389 (propositions en 1973) /260 (propositions en 1970) $=1,5$. On peut se débarrasser des décimales en multipliant par 100, et l'on obtient ainsi un indice d'interventionnisme relatif égalant:

$$
\frac{\mathrm{N}^{\mathrm{PQ}}{ }_{\mathrm{t}+\mathrm{x}}}{\mathrm{N}^{\mathrm{PQ}}{ }_{1970}}(100) \text { pour le PQ et } \frac{\mathrm{N}^{\mathrm{PLQ}}{ }_{\mathrm{t}+\mathrm{x}}}{\mathrm{N}^{\mathrm{PLQ}}{ }_{1970}}(100) \text { pour le PLQ }
$$

Un indice de comparaison interpartisane peut être calculé de la façon suivante: $\frac{\mathrm{N}^{\mathrm{PQ}}{ }_{\mathrm{t}+\mathrm{x}}}{\mathrm{N}^{\mathrm{PLQ}}{ }_{1970}}$ (100) pour comparer la propension à l'inteventionnisme du PQ à celle du PLQ. Le graphique 1 illustre les résultats du calcul de ces indices. La propension à l'interventionnisme du PLQ et du PQ curieusement similaire. L'indice d'interventionnisme relatif du PLQ démarre de 100 (pour 
260 propositions) en 1970 pour faire un bond à 150 en 1973 , puis s'accroître de seulement 23 points de 1973 à 1976, avant de faire un saut à 343 en 1981, pour finalement redescendre à seulement 141 points à l'élection de 1985. L'indice d'interventionnisme relatif du PQ évolue à un rythme très semblable: de 100 (pour 355 propositions) en 1970, il grimpe à 165 en 1973, puis à 185 en 1976, pour atteindre le sommet de 333 points en 1981, avant de chuter à 256 en 1985. L'évolution de l'indice d'interventionnisme relatif est similaire à plusieurs égards chez les deux partis: gain d'une cinquantaine de points de 1970 à 1973, puis d'une vingtaine de points de 1973 à 1976, suivi d'un bond d'environ 150 points de 1976, enfin, par la suite, d'une chute de plus de 200 points dans l'indice du PLQ, mais de seulement 77 points dans le cas du PQ. La période 1976-1985 ressort clairement comme une période de rupture: les partis démontrent une propension à l'interventionnisme beaucoup plus forte de 1976 à 1981 qu'au cours de la période précédente, tandis que la période 1981-1985 marque le déclin de la propension interventionnisme des partis. Ce revirement idéologique a été moins marqué dans les programmes du PQ que dans ceux du PLQ.

\section{GRAPHIQUE 1}

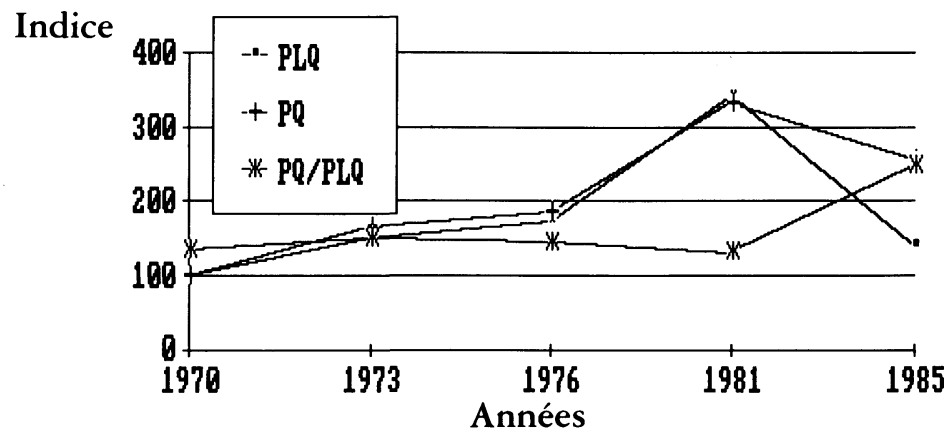


L'indice de comparaison interpartisane (PQ/PLQ) montre toutefois que si l'on compare le nombre de projets d'actions d'un parti à l'autre en supposant que les 260 projets formulés par le PLQ en 1970 équivalent à 100 points, l'indice d'interventionnisme relatif comparé du PQ s'élève à 136 points en 1970, 224 en 1973, 252 en 1976, 454 en 1981 et 350 en 1985. Les deux partis évoluent donc sur une trajectoire parallèle dans laquelle l'interventionnisme est toujours plus accentué chez le PQ que du côté du PLQ. Considéré sous cet angle la distance entre les deux partis atteint son minimum en 1970 et son maximum en 1981.

En résumé, les fluctuations relatives de la propension à l'interventionnisme ont été plus faibles dans les programmes du PQ que dans ceux du PLQ. D'autre part, la propension à l'interventionnisme a toujours été plus accentuée chez le PQ que dans les programmes libéraux. Le PLQ et le PQ ne compétionnent donc pas sur le mode de la confrontation directe mais sur celui de l'accentuation sélective.

\section{L'orientation de l'intervention de l'État}

En dépit de la richesse d'informations qu'il recèle, l'indice d'interventionnisme relatif véhicule une lacune sérieuse: il ne révèle rien sur l'orientation de l'interventionnisme des partis. Mellos (1970) et Latouche (1976) ont résolu ce problème en construisant des indices d'orientations idéologiques fondés sur des dichotomies définissant la position des propositions d'actions des partis. L'étude de Pelletier (1980) aborde elle aussi l'orientation idéologique de l'interventionnisme en classant les propositions d'actions des partis en fonction de quelques grandes dichotomies. L'indice de globalisme idéologique de Latouche regroupe les dichotomies spécifique-général, substantiel-formel et pragmatiqueidéologique. Dans l'étude de Latouche, une propositon était considérée quand elle renvoyait à une action spécifique ou à la création 
d'un organisme dont on précisait les fonctions et, elle était jugée comme générale dans les autres circonstances. D'autre part, une proposition était codée substantielle si elle comportait un effet direct et immédiat sur le public, ou elle était codée formelle si elle se limitait à envisager la création de structures formelles. Enfin, une proposition prévoyant une action politique précise en réponse à une situation spécifique était classée comme pragmatique, alors que les énoncés de principes étaient considérés comme des énoncés idéologiques.

Par ailleurs l'orientation de l'interventionnisme de l'État peut aussi être évaluée en fonction de la dichotomie classique public-privé. Une proposition d'action est jugée privée si elle est favorable à l'entreprise privée ou prévoit des crédits additionnels pour le secteur privé, mais elle est considérée comme publique si elle favorise le secteur public ou prévoit un contrôle étatique accru sur des secteurs de l'économie. D'après Latouche, les propositions d'actions du PLQ sont plus spécifiques, plus formelles et plus idéologiques que celles du PQ. Inversement les projets du PQ sont plus généraux, plus substantiels et plus pragmatiques que ceux des libéraux.

L'indice de Pelletier (1980) se fonde aussi sur la dichotomie privé-public. Pour ce dernier, l'interventionnisme renvoie à toute proposition favorisant le secteur public, alors que toute proposition favorable au secteur privé ou limitant le rôle du secteur public est considéré comme non interventionniste. La même étude considère que toute mesure de distribution ou de redistribution de prestations directes de biens et services aux individus définit de l'interventionnisme à orientation individualiste, alors que l'interventionnisme à orientation collectiviste se définit par des mesures favorables à la collectivité qui ne se concrétisent pas dans la prestation directe de biens et services aux individus. L'application de ces dichotomies aux programmes des partis amène Pelletier à conclure, qu'au cours de la période 1970-1976, le PQ est à la fois plus interventionniste 
et plus interventionniste à orientation collectiviste que le PLQ, ce dernier tendant à devenir moins interventionniste avec le temps.

L'idée implicite, qui sous-tend les études empiriques de Latouche et Pelletier, est que les propositions d'actions des programmes des partis offrent des biens et des services comportant des bénéfices plus ou moins concrets pour les électeurs. L'ouvrage magistral d'Olson (1965) sur La logique de l'action collective, a démontré que les caractéristiques des biens collectifs offerts par les groupes d'intérêt affectent les individus dans leurs décisions de joindre un groupe et de participer à ses activités de production de biens collectifs. L'ambigüité de la conception olsonnienne au sujet des biens collectifs a toutefois soulevé de nombreuses critiques. Contrairement à Olson, qui démontre qu'il n'est pas rationnel de participer à un groupe qui produit des biens publics purs, Chamberlin (1974) a soutenu qu'il est rationnel de contribuer à un groupe qui produit des biens publics purs non susceptibles de congestion qui sont supérieurs en termes de demande. En outre, Moe (1981) a prouvé mathématiquement qu'un individu rationnel pouvait être amené à participer à un groupe offrant uniquement des biens publics purs. Les études empiriques de Marsh (1976) sur la Confederation of British Industry, de Tillock et Morrison (1979) sur le Zero Population Growth Movement et de Cook (1984) sur quatre groupes américains d'intérêt public ont démontré que les individus étaient beaucoup plus sensibles que prévu aux bénéfices que comportent les biens publics purs et qu'il importait donc de disposer d'une conception des biens collectifs beaucoup plus précise que celle d'Olson.

L'ambigüité de la conception olsonnienne au sujet des biens collectifs peut être levée en consultant les travaux classiques d'économistes, tels que Samuelson (1956) et Head (1972). Plus récemment, des politistes tels que Ostrom $(1977,1983,1986)$ et Sproule-Jones (1983), notamment, ont démontré à nouveau l'importance que revêtent les caractéristiques des biens collectifs dans 
les décisions des individus. Ces développements théoriques et mathématiques n'ont toutefois pas encore débouché sur des applications empiriques dans le cas des propositions d'actions des programmes des partis politiques.

À l'instigation de la plupart des économistes et des politistes qui ont abordé l'analyse de la production et de la consommation des biens collectifs sous l'angle théorique, on distinguera les biens collectifs en fonction de deux critères intrinsèques: la possibilité d'exclusion et la possibilité de division des bénéfices offerts. L'exclusion aux bénéfices d'une proposition d'action peut être jugée facile si un destinataire potentiel des bénéfices de cette proposition d'action gouvernementale peut être empêché de recevoir les bénéfices en question parce qu'il ne satisfait pas un critère ou une condition explicite déterminée dans le programme d'un parti. À l'inverse, l'exclusion aux bénéfices d'un projet d'action gouvernementale peut être considérée comme difficile lorsqu'aucun destinataire potentiel ne peut être empêché de recevoir les bénéfices offerts parce que le programme d'un parti ne prévoit pas de critères explicites d'exclusion ou est incapable d'en imposer. Cette éventualité se produit lorsqu'il est impossible d'exclure des destinataires potentiels pour des raisons techniques parce qu'il est impossible de contrôler l'accès aux bénéfices d'une intervention gouvernementale, et pour des raisons économiques liées au caractère prohibitif des coûts d'exclusion, comme ce serait le cas, par exemple, pour la réduction du niveau de pollution de l'air.

Par ailleurs les bénéfices d'une proposition d'intervention gouvernementale peuvent être considérés comme divisibles dans les cas où ils totalisent une somme finie qui peut être fractionnée entre des destinataires potentiels. Dans le cas contraire, c'est-àdire lorsqu'il est impossible d'en établir la somme, les bénéfices d'une proposition d'action gouvernementale sont considérés comme indivisibles. La dichotomie divisible-indivisible renvoie au même concept que les dichotomies substantiel-formel, spécifique-général 
et pragmatique-idéologique. À la différence de ces dichotomies, la dichotomie divisible-indivisible renvoie explicitement plutôt qu'implicitement aux bénéfices que peuvent recevoir les électeurs.

La combinaison des dichotomies exclusion facile-difficile et bénéfices divisibles-indivisibles permet de distinguer quatre types de biens collectifs.

\section{TABLEAU 1}

Quatre types de biens collectifs

\begin{tabular}{|l|l|l|}
\cline { 3 - 3 } \multicolumn{2}{c|}{} & \multicolumn{1}{l|}{$\begin{array}{l}\text { divisibilité des bénéfices d'une proposition } \\
\text { d'intervention gouvernementale }\end{array}$} \\
\hline $\begin{array}{l}\text { exclusion } \\
\text { aux bénéfices } \\
\text { d'une proposition } \\
\text { d'intervention } \\
\text { gouvernementale }\end{array}$ & facile & divisibles indivisibles \\
\cline { 2 - 4 } & difficile & biens quasi publics biens quasi privés \\
\cline { 2 - 3 } & & biens publics purs \\
\hline
\end{tabular}

Il n'est peut être pas inutile d'illustrer à l'aide de quelques exemples le résultat de l'utilisation de cette double dichotomie:

Biens privés purs

- PLQ aidera par une assistance financière spéciale les entreprises qui réussiront à stabiliser leur emploi.

- PQ interviendra afin de procurer de l'emploi aux travailleurs handicapés.

- PQ mettra sur pied des services d'accueil à l'intention des femmes battues.

\section{Biens quasi privés}

- PLQ instaurera un congé de maternité sans perte de droits acquis pour les travailleuses enceintes. 
- PQ permettra au travailleur de prendre sa retraite à 55 ans s'il le veut tout en l'assurant de travailler jusqu'à un âge avancé.

- PQ permettra aux propriétaires d'une entreprise familiale non incorporée de déduire le salaire du conjoint comme dépense d'exploitation.

\section{Biens quasi publics}

- PQ fournira l'assistance financière nécessaire aux fins de restauration et de préservation.

- PLQ, animateur plutôt qu'initiateur, soutiendra les groupes qui veulent agir et innover dans les domaines sociaux.

- PLQ mettra sur pied les infrastructures nécessaires à l'intégration de l'industrie de la pêche par la création de parcs industriels.

\section{Biens publics purs}

- PLQ définira une politique de valorisation et d'enrichissement de la fonction de travail qui tienne compte de la dimension des relations de travail.

- PQ améliorera la qualité de la vie, préservera notre environnement.

- PQ visera à la libération collective et à l'épanouissement individuel de tous les travailleurs.

$\mathrm{Si}$, comme le soutiennent Olson et les spécialistes de la théorie des biens collectifs, les électeurs sont sensibles aux caratéristiques intrinsèques des bénéfices des biens offerts, les partis politiques tenteront d'exploiter cette situation en promettant plus ou moins de biens privés et de biens publics, suivant qu'ils estiment que les électeurs sont sensibles à l'obtention de bénéfices spécifiques, substantiels et pragmatiques (c'est la perspective olsonnienne), ou à l'obtention de bénéfices généraux, formels et idéologiques (c'est la perspective de Moe). 
Les analystes et les commentateurs de la scène politique québecoise tendent généralement à associer le Parti libéral du Québec au spécifique, au substantiel et au pragmatique Parti québécois au général, au formel et à l'idéologique, postulant plus ou moins explicitement que le PLQ tente de gagner des votes au moyen de propositions d'interventions gouvernementales comportant des bénéfices spécifiques, substantiels et pragmatiques alors que le $\mathrm{PQ}$ poursuit le même objectif par le recours à des propositions d'interventions gouvernementales comportant des bénéfices généraux, formels et idéologiques. Dès lors, on peut faire deux hypothèses: 1) les programmes d'actions du PLQ comportent une plus grande proportion de propositions de biens privés que de biens publics; 2) le PQ promet une plus grande proportion de propositions de biens publics que le PLQ. Qu'en est-il dans les faits?

\section{GRAPHIQUE 2}

Pourcentage moyen des propositions d'interventions gouvernementales du PLQ et du PQ selon les types de biens offerts pour l'ensemble de la période 1970-1985
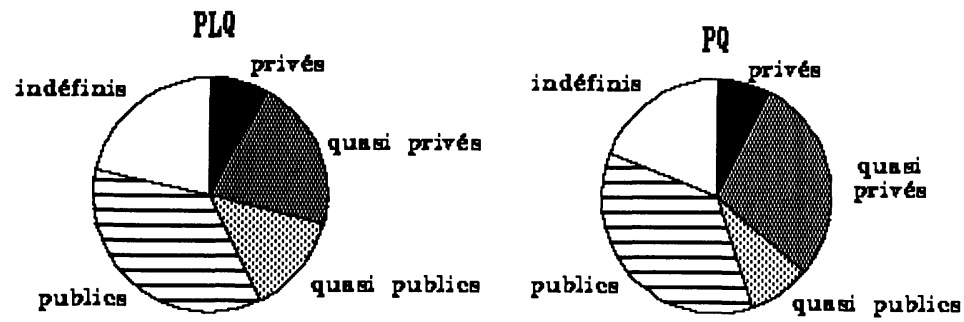

Types de biens

$P L Q$

$\%$

privés

quasi privés

quasi publics

publics

indéfinis

$\begin{array}{rr}8,1 & 7,6 \\ 20,8 & 28,6 \\ 13,7 & 8,7 \\ 35,7 & 36,2 \\ 21,6 & 18,8\end{array}$


Considérant globalement l'ensemble des programmes de 1970 , 1973, 1976, 1981 et 1985, le panier de propositions d'actions du PLQ est composé de 8,1\% de projets de biens privés, 20,8\% de biens quasi privés, $13,7 \%$ de biens quasi publics et $35,7 \%$ de biens publics. Un peu plus du cinquième des projets d'actions du PLQ a été classé dans la catégorie des biens indéfinis, soit des propositions d'interventions gouvernementales ne comportant pas suffisamment d'informations explicites pour déterminer le degré d'exclusion ou le degré de divisibilité des bénéfices promis (graphique 2). Quant au PQ, son panier comprend 7,6\% de biens privés, $28,6 \%$ de biens quasi privés, $8,7 \%$ de biens quasi publics, $36,2 \%$ de biens publics et $18,8 \%$ de biens indéfinis. En moyenne, les partis different assez peu, si ce n'est que le panier de projets du PQ comporte une plus grande proportion de biens quasi privés que le PLQ et que celui-ci tend à promettre une plus grande proportion de biens quasi publics que le PQ. Un examen global des projets d'actions qui compare les paniers de projets d'actions des partis ne confirme donc pas les deux hypothèses formulées cihaut.

L'examen de l'évolution des projets d'interventions gouvernementales des partis montre que des changements importants sont survenus depuis 1970 (tableau 2). Si l'on fait exception des programmes de 1970 et de 1981, le PLQ a toujours promis aux électeurs une plus faible proportion de biens privés que le PQ. Les programmes de 1981 marquent un point de rupture chez les deux partis, le PLQ promettant alors 5,6\% de plus de biens privés qu'en 1976 et 5,1\% de plus qu'en 1985, alors que le PQ en promet 0,8\% de moins qu'en 1976 et 3,7\% de moins qu'en 1985. L'écart des programmes de 1981 n'est pas facile à expliquer: on aurait pu penser, qu'après l'échec référendaire de 1980, le PQ aurait pu tenter de regagner la faveur des électeurs en leur promettant une plus grande proportion de bénéfices tangibles qu'en 1976; d'autre part, on aurait pu penser que, sous la gouverne de $\mathbf{M}$. 
Claude Ryan, le PLQ aurait accordé moins d'importance que par le passé aux promesses de bénéfices privés. Les programmes de 1981 constituent donc des programmes de déviation qui défient toute tentative d'explication simple. La place relative qu'occupe les promesses de bénéfices privés dans les programmes du PLQ tend à être relativement stable alors qu'elle tend à s'accroître du côté des programmes péquistes. Contrairement à toute attente, le parti qui tend à accorder de plus en plus d'importance aux propositions d'actions engendrant des bénéfices privés n'est pas le Parti libéral mais le Parti québécois. Ces résultats montrent que la compétition pour les votes ne se joue pas sur le mode de la confrontation directe puisque les partis modifient leurs positions de façon parallèle, le PQ mettant davantage l'accent sur les bénéfices privés.

Une hypothèse un peu plus faible consiste à stipuler que le PLQ tente davantage que le PQ de gagner les votes des électeurs en leur promettant des bénéfices quasi privés dans ses programmes. Cette hypothèse est contredite par les faits de façon décisive puisque, au fil du temps, le PLQ tend à accorder de moins en moins de place aux projets de bénéfices quasi privés alors que le PQ tend, au contraire, à leur attribuer une place relative de plus en plus grande. En 1985, le programme du PLQ était constitué de 16,3\% de projets comportant des bénéfices quasi privés contre $32,9 \%$ de projets similaires dans le programme du PQ. À ce chapitre, la compétition entre les partis se déroule donc sur le mode de la confrontation directe plutôt que sur celui de l'accentuation sélective puisque les positions des partis tendent de plus en plus à diverger.

Une autre hypothèse caractérisant l'orientation de l'interventionnisme des partis était que les programmes du $P Q$ accordent une importance relative plus élevée que le PLQ aux bénéfices publics. Cette hypothèse est confirmée par les faits pour la période 1970-1976, mais non fondée pour le reste de la période. Ainsi, le PQ a accordé une place relative plus importante que le PLQ 
TABLEAU 2

Pourcentage des propositions d'interventions gouvernementales des programmes du PLQ et du PQ selon les types de biens collectifs offerts pour la période 1970-1985

\begin{tabular}{|c|c|c|c|c|c|}
\hline & 1970 & 1973 & 1976 & 1981 & 1985 \\
\hline \multicolumn{6}{|l|}{ biens privés purs } \\
\hline PLQ & 8,3 & 6,2 & 6,7 & 12,3 & 7,2 \\
\hline $\mathrm{PQ}$ & 4,1 & 7,8 & 8,1 & 7,3 & 11,0 \\
\hline \multicolumn{6}{|l|}{ biens quasi privés } \\
\hline PLQ & 14,9 & 28,1 & 22,5 & 22,4 & 16,3 \\
\hline PQ & 27,5 & 26,5 & 26,4 & 29,9 & 32,9 \\
\hline \multicolumn{6}{|l|}{ biens quasi publics } \\
\hline PLQ & 16,2 & 16,8 & 15,3 & 11,8 & 8,3 \\
\hline PQ & 4,4 & 10,2 & 10,4 & 9,0 & 9,4 \\
\hline \multicolumn{6}{|l|}{ biens publics purs } \\
\hline PLQ & 35,7 & 33,2 & 34,3 & 38,1 & 37,1 \\
\hline$P Q$ & 42,5 & 36,0 & 36,2 & 35,9 & 30,5 \\
\hline \multicolumn{6}{|l|}{ biens indéfinis } \\
\hline PLQ & 24,9 & 15,7 & 21,1 & 15,5 & 31,0 \\
\hline$P Q$ & 21,6 & 19,5 & 18,9 & 17,9 & 16,3 \\
\hline \multicolumn{6}{|l|}{ Total } \\
\hline PLQ & $\begin{array}{c}100 \\
(260)\end{array}$ & $\begin{array}{c}100 \\
(389)\end{array}$ & $\begin{array}{c}100 \\
(450)\end{array}$ & $\begin{array}{c}100 \\
(893)\end{array}$ & $\begin{array}{c}100 \\
(367)\end{array}$ \\
\hline PQ & $\begin{array}{c}100 \\
(355)\end{array}$ & $\begin{array}{c}100 \\
(584)\end{array}$ & $\begin{array}{c}100 \\
(656)\end{array}$ & $\begin{array}{c}100 \\
(1181)\end{array}$ & $\begin{array}{c}100 \\
(910)\end{array}$ \\
\hline
\end{tabular}

aux bénéfices publics en 1970, 1973 et 1976, alors qu'en 1981 et 1985 , c'est le PLQ qui a attribué le plus de place relative aux propositions d'interventions gouvernementales comportant des bénéfices publics. Plus encore, les programmes du PQ tendent à laisser de moins en moins de place aux bénéfices publics au fil des ans, alors que le PLQ évolue dans le sens contraire, accordant de plus en plus d'espace aux bénéfices publics. Le parti politique qui accorde l'importance relative la plus élevée aux bénéfices axiologiques ou symboliques n'est donc pas le Parti québécois mais 
le Parti libéral. Contrairement aux prétentions de plusieurs, le parti qui tente de se vendre avec des grands principes n'est plus le PQ mais le PLQ. La compétition entre les partis se déroule donc ici aussi sur le mode de la confrontation, les positions de chacun, sur l'importance à accordèraux bénéfices publics tendant de plus en plus à diverger au fil des ans.

Une hypothèse un peu plus faible consiste à supposer que le PQ accorde une plus grande place que le PLQ aux bénéfices quasi publics. Comme l'hypothèse concernant les biens publics, cette hypothèse est elle aussi en grande partie contredite par les faits. À l'exception du programme de 1985, où le PLQ a accordé moins de place aux bénéfices quasi publics que le PQ, le PLQ a toujours attribué plus d'espace aux propositions d'interventions gouvernementales comportant des bénéfices quasi publics que ne l'a fait le PQ. L'évolution de la position des partis sur les bénéfices quasi publics est assez claire: le PQ leur accorde une place relative qui varie entre 9 et $10 \%$ tandis que de 1970 à 1985, la part de ces bénéfices a chuté de 16 à $8 \%$ du côté du PLQ. Au chapitre des bénéfices quasi publics, la compétition entre les partis se joue sur le mode de l'accentuation sélective, le Parti libéral tendant à mettre de moins en moins d'emphase sur ces bénéfices.

\section{Conclusion}

L'analyse comparée de l'orientation de l'interventionnisme dans les idéologies du Parti libéral du Québec et du Parti québécois depuis 1970 permet d'arriver à plusieurs résultats qui contredisent partiellement le sens commun: 1) les fluctuations relatives de la propension à l'interventionnisme ont été plus faibles dans les programmes du PQ que dans ceux du PLQ; 2) la propension à l'interventionnisme a toujours été plus accentuée dans les programmes péquistes que dans les programmes libéraux; 3) contrairement à toute attente, les programmes qui tendent à 
accorder de plus en plus d'importance aux propositions d'actions comportant des bénéfices privés se trouvent dans les programmes du PQ plutôt que dans ceux des libéraux; 4) contrairement aux prétentions de plusieurs, le parti qui tente de se vendre en promettant des grands principes n'est plus le PQ mais le PLQ; 5) la compétition entre le PLQ et le PQ ne se joue pas toujours, comme l'avait déjà confirmé Latouche (1976), sur le mode de l'accentuation sélective mais aussi sur celui de la confrontation directe.

L'analyse comparée de l'orientation de l'interventionnisme dans les idéologies des partis montre que la recherche empirique permet d'arriver à des résultats qui contredisent la connaissance intuitive.

\section{BIBLIOGRAPHIE}

Barry, B., 1978, Sociologists Economists and Democracy, Chicago, University of Chicago Press.

Budge, I et D.J. Farlie, 1983 «Party Competition: Selective Emphasis or Direct Confrontation? An Alternative with Data", in H. Daalder et P. Mair, eds., Western European Party Systems, Beverly Hills, Ca., Sage Publications, pp. 267-305.

Budge, I. et D.J. Farlie, 1977, Voting and Party Competition, New York, Wiley.

Chamberlin, J., 1974, "Provision of Collective Goods as a Function of Group Size», American Political Science Review, LXVIII-2: 707-716.

Cook, C.E., 1984, «Participation in Public Interest Group. Membership Motivations», American Politics Quarterly, 12-4: 409-430.

Dion, L., 1975, Nationalisme et Politique au Québec, Montréal, Hurtubise, HMH.

Downs, A., An Economic Theory of Democracty, New York, Harper and Row, 1957.

Hansen, J.M., 1985, "The Political Economy of Group Membership", American Political Science Review, 79-1: 79-96.

Head, J.G., 1972, "Public Goods: The Polar Case», R.M. Bird et J.G. Head, eds, 1972, Modern Fiscal Issues: Essays in Honour of Carl S. Shoup, Toronto, University of Toronto Press, pp. 7-16.

Kiser, L. et E. Ostrom, 1982, "The Three Worlds of Action: A Metatheoretical Synthesis of Institutional Approaches", in Ostrom, E., ed, 1982, Strategies of Political Inquiry, Beverly Hills, Ca. Sage Publication, pp. 179-222.

Krippendorff, K.H., 1980, Content Analysis: An Introduction to Its Methodology, Beverly Hills, Ca., Sage Publications.

Landry, R., Duchesneau, P., 1987, «L'offre d'interventions gouvernementales aux groupes: une théorie et une application, Revue canadienne de science politique, XX-3: 525552. 
Latouche, D., 1976, «Le contenu thématique et l'orientation idéologique des programmes électoraux de 1973", dans D. Latouche, G. Lord et J.-G. Vaillancourt, Le processus électoral au Québec: les élections provinciales de 1970-1973, Montréal, HurtubiseHMH, pp. 127-150.

McRoberts, K. et D. Posgate, 1983, Développement et modernisation du Québec, Montréal, Boréal Express.

Marsh, D., "On Joining Interest Groups: An empirical Consideration of the Work of Mancur Olson Jr.", British Journal of Political Science, 6: July: 257-271.

Mellos, K., 1970, "Quantitative Comparison of Party Ideology», Revue canadienne de science politique, 111-3: 540-558.

Moe, T.M., 1981, "Toward a Broader View of Interest Groups", The Journal of Politics, 43-2: 531-543.

Moe, T.M., 1980, "A Calculus of Group Membership», American Journal of Political Science, 24-4: 593-632.

Monière, D., 1977, Le développement des idéologies au Québec, Montréal, Québec/Amérique.

Musgrave, R.A. et P.B. Musgrave, 1980, Public Finance in the Theory and Practice, Third edition, New York, McGraw Hill Book Company.

Olson, M. Jr., 1965, The logic of Collective Action. Public Goods and the Theory of Groups, New York, Schoken Books.

Ordeshook, P.C., 1976, "The Spatial Theory of Elections: A Review and a Critique" dans I. Budge, I. Crewe et D. Farlie (dir.), Party Identification and Beyond Representations of Voting and Party Competition, New York, Wiley, 1976.

Ostrom, E., 1983, "A Public Choice Approach to Metropolitan Institutions: Structure, Incentives, and Performance», The Social Science Journal, 20-3: 79-96.

Ostrom, E. 1986, «An Agenda for the Study of Institutions», Public Choice, 48-1: 326.

Ostrom, V et E. Ostrom, 1977, «Public Goods and Public Choice», p. 49 dans Savas, S. ed., 1977, Alternatives for Delivering Public Services, Boulder, Co., Westview Press.

Pelletier, R., 1980, «Les partis politiques et l'État», in G. Bergeron et R. Pelletier (dir.), L'État du Québec en devenir, Montréal, Boréal Express, pp. 241-262.

Pomper, G.M., et Lederman, S.S., 1980, Elections in America: Control and Influence in Democratic Politics, $2^{\mathrm{e}}$ éd., New York, Longman.

Riker, W.H. et P.C. Ordeshook, 1973, An Introduction to Positive Political Theory, Englewood Cliffs, N.J., Prentice Hall.

Rioux, M., 1968, "Sur l'évolution des idéologies au Québec», Revue de l'Institut de sociologie, no 1: 95-124.

Robertson, D. 1976, A Theory of Party Competition, New York, John Wiley.

Rose, R., 1984, Do Parties Make a Difference?, Second Edition, London, MacMillan.

Salisbury, R.H., 1984, «Interest Representation: The Dominance of Institutions", American Political Science Review, 78-1: 64-7.

Salisbury, R.H., 1983, "Interest Groups: Toward a New Understanding", dans Cigler, A.J. et B.A. Loomis, eds, 1983, Interest Group Politics, Washington, CQ Press, pp. 354-369.

Salisbury, R.H., 1969, "An Exchange Theory of Interest Groups", Midwest Journal of Political Science, 13-1: 1-32. 
Samuelson, P., 1954, «The Pure Theory of Public Expenditure», Review of Economics and Statistics, 36: 387-389.

Sproule-Jones, M., 1983, "Institutions, Constitutions and Public Policies: A PublicChoice Approach" dans M.M. Atkinson et M.A. Chandler, eds., The Polities of Canadian Public Policy, Toronto, The University of Toronto Press, pp. 127-150.

Tillock, H. et D.E. Morrison, 1979, "Group Size and Contributions to Collective Action: An Examination of Olson's Theory Using Data from Zero Population Growth, Inc", Research in Social Movement, Conflits and Change, Vol. 2: 131-158.

Walker, J.L., 1983, "The Origins and Maintenance of Interest Groups in America», American Political Science Review, 77-2: 390-406. 